\title{
Urticaria pigmentosa: A rare case report
}

\author{
Krishnendra Varma ${ }^{1}$, Noreen Munshi ${ }^{2, *}$, Ujjwal Kumar $^{3}$ \\ ${ }^{1}$ Professor \& HOD, ${ }^{2}$ PG Student, ${ }^{3}$ Associate Professor, Dept. of Dermatology, Ruxmaniben Deepchand Gardi Medical College, \\ Ujjain, Madhya Pradesh, India
}

*Corresponding Author:

Email: noreenmunshi10@hotmail.com

\begin{abstract}
Mastocytosis is a rare, acquired multisystem disorder of increased number of mast cell in the skin and other tissues. Mastocytosis is caused by several activating mutations in the KIT gene. ${ }^{1}$ Although mastocytosis is primarily a systemic disorder, it often presents with skin lesions to dermatologists. Urticaria Pigmentosa is the most common pattern of cutaneous mastocytosis in adults and children.
\end{abstract}

Keywords: Urticaria Pigmentosa, Mastocytosis.

\section{Introduction}

Mastocytosis is a rare, acquired disorder characterized by an abnormal increase of mast cells and release of cell mediators in the skin and other tissues. The incidence is 1:10000 to 1:30000 including children and adults. Mastocytosis has been classified by WHO into: cutaneous and systemic mastocytosis. Urticaria pigmentosa is the most common type (70-90\%) of cutaneous mastocytosis. Urticaria pigmentosa developed in the first year of life in $84 \%$ of 67 children [2]; the most common age of onset for the disease in adults is between 20-40 years. When other organs of the body such as bone marrow, lymph nodes, liver, gut and spleen are affected the disorder is called systemic mastocytosis.

\section{Case Report}

A 10-year-old female, presented with multiple reddish brown lesions, which started over the face and further progressed to involve the upper limbs and trunk since past 2 years. She gave a history of elevation and redness, associated with itching on the lesions after a few minutes of rubbing. There were no associated systemic symptoms. The personal and family history was not contributory.

On examination, there were multiple hyper pigmented macules over the forehead, face, neck, upper limbs and trunk. The lesions urticatiated within few minutes of gentle rubbing suggestive of the Darier's sign, were associated with itching and subsided within 15-20 minutes.

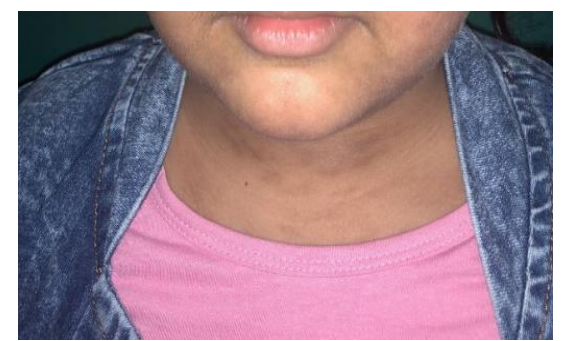

Fig. 1: Hyperpigmented macules over the neck and chin

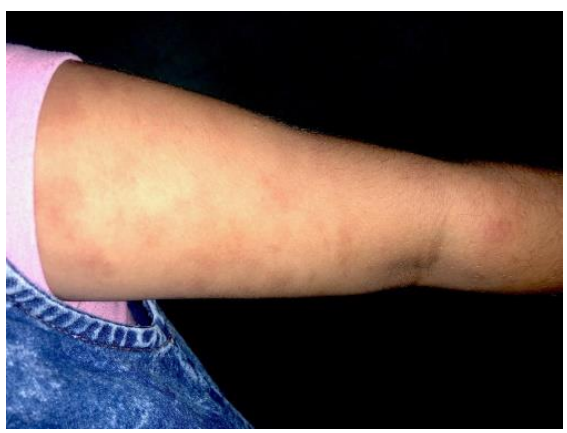

Fig. 2: Hyperpigmented macules over the Left upper limb

A complete blood count, liver and renal function tests and urine analysis were within normal limits. The biospy of a macular lesion from the left arm showed increased melanisation of the epidermis with rounded mast cells infiltrating the dermis. The mast cells were identified using the toluidine blue stain. The histopathological findings were suggestive of urticaria pigmentosa.

The treatment was mostly symptomatic. The patient was given oral antihistamines and PUVA therapy and was provided reassurance about the course and prognosis of the disease. Management relies on avoidance of trigger factors for mast cell degranulation, symptomatic relief with anti-mast cell mediator drugs, immunotherapy when appropriate (e.g. venom allergy) and early detection of systemic disease to avoid further complications. 


\section{Conclusion}

Urticaria pigmentosa is the commonest form of mastocytosis with onset usually below two years of age. The lesions are reddish-brown, maculopapules, plaques or nodules. The urticaria pigmentosa diagnosis is mainly clinical, with a positive Darier's sign, which is pathognomonic and is positive in $90 \%$ of cases. In certain cases, a skin biopsy is required to confirm the diagnosis. Children with the disease do not require extensive investigations if they are well. The lesions usually resolve spontaneously before adolescence but some cases may persist into adult life. Even though systemic involvement is possible, malignant transformation is extremely rare but, can occur in some cases. No cures are currently available but there is increasing evidence of the benefit of cytoreductive therapies in patients with organ dysfunction or failure.
Funding: No funding sources.

\section{Conflict of interest: None declared.}

\section{References}

1. Macri A, Cook C. Urticaria Pigmentosa (Cutaneous Mastocytosis) [Updated 2018 Feb 14]. In: StatPearls [Internet]. Treasure Island (FL): StatPearls Publishing; 2018 Jan-.Available from: https://www.ncbi.nlm.nih.gov/books/NBK482503/

2. Azaña JM, Torrelo A, Mediero IG, Zambrano A. Urticari a pigmentosa: a review of 67 pediatric cases. Pediatr Dermatol 1994;11:102-6.

How to cite this article: Varma K., Munshi N., Kumar U. Urticaria pigmentosa: A rare case report. Indian J Clin Exp Dermatol 2018;4(4):348-49. 